\title{
CURRENT STATUS OF GEOPHYSICAL MODELS OF NUTATION
}

\author{
P. M. MATHEWS \\ Harvard-Smithsonian Center for Astrophysics \\ Cambridge, Massachusetts
}

AND

V. DEHANT

Royal Observatory of Belgium

Brussels, Belgium

Geophysical modeling of nutations involves (a) the formulation of a general theory of the forced nutations of an idealized Earth (spheroidal, oceanless, elastic), supplemented by separate theoretical treatments of the effects of the oceans, anelasticity, and other deviations from the ideal, and (b) fitting of the theoretical expressions to VLBI-estimated amplitudes of the various spectral components of the nutations. The aim is to obtain best-fit estimates of Earth parameters which influence the nutations; and the degree of success of the modeling is judged by the precision of the estimates obtained, as well as the quality of the fit as measured by $\chi_{r e d}^{2}$, the chisquared per degree of freedom.

We present here our findings from two types of fits using, for the observational input, the nutation amplitude estimates for seven important pairs (retrograde and prograde) of circular nutations, together with their standard errors and mutual correlations, which were kindly furnished by T.A. Herring (private communication, 1993). The theoretical framework employed was that of Mathews et al. (1991), hereinafter referred to as MBHS, together with the Zhu and Groten (1989) rigid Earth nutation series modified to take account of the recently-estimated correction of -0.3 $m a s / c y$ to the precession constant. Estimates of ocean tide effects, and of anelasticity effects based on Wahr and Bergen's (1986) results for model QMU with $\alpha=0.15$ (hereafter abbreviated to WB), were taken from Herring et al. (1991). Alternative estimates of anelasticity effects (DZ, due to Dehant (1990) based on the Zschau model), were also considered. 
The theory of MBHS shows how the amplitude $\tilde{\eta}(\sigma)$ of the circular nutation due to a tidal component of frequency $\sigma$ in cpsd and amplitude $\tilde{\phi}(\sigma)$ is determined by certain Earth parameters: the ratios of moments of inertia, the ellipticities, and the compliances (deformability parameters), of the Earth and of its outer and inner core regions. It turns out that $\tilde{\eta}(\sigma) / \tilde{\phi}(\sigma)$ is governed primarily by $e$ and three composite parameters $p_{i}$ :

$$
p_{1}=\left(A / A_{m}\right)(e-\kappa), \quad p_{2}=\left(A / A_{m}\right)\left(e_{f}-\beta\right), \quad p_{3}=\left(A_{f} / A_{m}\right)(e-\gamma)
$$

where $e, e_{f}$ are the respective dynamic ellipticities and $A, A_{f}$ the moments of inertia, of the Earth and of its fluid core, $A_{m} \approx\left(A-A_{f}\right)$ is the moment of inertia of the mantle, and the remaining parameters are compliances. Among the parameters other than the above which enter into the theory, the ellipticity $e_{s}$ of the solid inner core seems to be the only one which could differ substantially from standard Earth models, there being no strong constraints on it from other considerations. One type of fit that we report on (Fit I) makes direct use of the above theory to fit the real (in-phase) parts of the observed amplitudes by a nonlinear least squares procedure, with estimation of the $p_{i}$ as functions of the assumed value of $e_{s}$.

The main findings are as follows: (i) For all assumed values of the inner core ellipticity $e_{s}$, ranging from less than one-eighth to over three times the value (0.00242) for Earth model PREM, $\chi_{r e d}^{2}$ remains practically unchanged by the application of corrections for ocean tide effects or for anelasticity using the WB estimates. But the estimates obtained for $p_{2}$ and $p_{3}$ depend strongly on the corrections applied; only when both the corrections are applied do they come close to being within reasonable range of the values computed from Earth models and to being consistent with inferences from other sources regarding the value of the $k_{2}$ Love number (to which $\kappa$ is proportional). A detailed examination suggests that improvement of ocean tide modeling may be the key to achieving greater consistency. (ii) The use of DZ instead of WB anelasticity estimates worsens the fit significantly: $\chi_{\text {red }}^{2}$ increases by over $60 \%$. (iii) Irrespective of the corrections applied, a low value for the inner core ellipticity is favoured by the data: $\chi_{\text {red }}^{2}$ is found to have a pronounced minimum at $e_{s} \approx 0.5 e_{s(P R E M)}$. The estimate for $p_{1}$ (which is only mildly altered by the application of the corrections) requires that $e_{f}$, and hence the $\mathrm{CMB}$ flattening, be higher than for the hydrostatic equilibrium Earth; the excess flattening required decreases as $e_{s}$ is increased, but is close to $450 \mathrm{~m}$ (within the range first inferred by Gwinn et al. (1986)) for $e_{s}$ between $e_{s(P R E M)}$ and the value favoured by our least squares fit. $(i v)$ If the 18.6 year nutations are excluded from the fit, $\chi_{\text {red }}^{2}$ gets reduced by a factor of two or more, and becomes nearly insensitive to the value of $e_{s}$. 
The last observation highlights the need for efforts to bring the theoretical and observational estimates of the 18.6 year nutations into much better agreement than at present. The role of these nutations in constraining the inner core ellipticity provides an extra incentive for such efforts. Discrimination between different models of anelasticity, and the role that Love number estimates (from satellite tracking data, for example) could play in assessing the degree of agreement between nutation theory and observations, are other interesting points emerging from our analysis.

The second type of fitting (Fit II) was aimed at estimating the parameters in a resonance formula of the MBHS form. If $\tilde{\eta}(\sigma)$ is normalized relative to the corresponding amplitude $\tilde{\eta}_{R}(\sigma)$ for a rigid Earth model, the normalized amplitude $\tilde{\eta}_{N}(\sigma) \equiv \tilde{\eta}(\sigma) / \tilde{\eta}_{R}(\sigma)$ has the resonance expansion

$$
\tilde{\eta}_{N}(\sigma)=1+R_{0}+R^{\prime}(1+\sigma)+\sum_{\alpha} \frac{R_{\alpha}}{\sigma-\sigma_{\alpha}},
$$

where the $\sigma_{\alpha}$ represent the eigenfrequencies of normal modes. (The relevant ones are: the Chandler Wobble, and the nearly diurnal wobbles associated with ( $a$ ) the free core nutation (FCN) and (b) the prograde free core nutation (PFCN) which arises from the presence of the solid inner core.) The rigid Earth amplitude is

$$
\tilde{\eta}_{R}(\sigma)=\frac{e}{e-\sigma} \tilde{\phi}(\sigma)
$$

where $\tilde{\phi}(\sigma)$ represents the amplitude of the relevant spectral component of the tidal potential. Fixing the values of the CW and PFCN parameters, we made least squares estimations of $R$ and $R^{\prime}$ along with the eigenfrequency and strength of the FCN mode (a) as complex parameters, to fit the complex nutation amplitudes and $(b)$ as real parameters, to fit the in-phase parts only. (The parameters are necessarily real for the idealized Earth, but acquire small imaginary parts due to ocean tide, anelasticity, and other dissipative processes.) Unlike in earlier estimations (e.g., Defraigne et al., 1994), it is possible here to get an idea of the influence of the inner core on the quality of the fit, by varying the input values of the PFCN parameters.

The main results are as follows: $(i)$ Irrespective of the corrections applied, the fit to the complex amplitudes is much poorer than to the real part alone; special efforts are clearly needed to bring observational and theoretical estimates of the out-of-phase part into agreement. (ii) As in Fit I, application of ocean tide and anelasticity (WB) corrections hardly changes $\chi_{r e d}^{2}$. But it makes the size of the imaginary parts of the parameters much smaller, as should be expected. The resulting increase in the estimated $Q$ of the FCN $\left(Q=\Re \sigma_{F C N} /\left(2 \Im \sigma_{F C N}\right)\right.$, where $\Re$ and $\Im$ mean real and imaginary parts) is from 16900 to 25300 . (iii) If the PFCN term is dropped altogether, 
the fit obtained is significantly better than when MBHS values are used for the PFCN parameters, suggesting that coupling of the inner core to the rest of the Earth is either much weaker or much stronger than for standard Earth models. The former alternative is consistent with the low value of $e_{s}$ favoured by Fit I. ( $i v$ ) Estimates from 429.3 to 431.5 days are obtained for the FCN period, depending on what corrections are applied, whether a complex or real fit is done, and whether the PFCN is retained or dropped.

The fit to the real parts alone is much better $\left(\chi_{\text {red }}^{2} 50 \%\right.$ smaller $)$ with Fit II than with Fit I. However the parameter estimates obtained with Fit II violate the sum rules

$$
R-\frac{R_{\alpha}}{\sigma-\sigma_{\alpha}}=1 ; \quad R+R^{\prime}(1+e)+\frac{R_{\alpha}}{e-\sigma_{\alpha}}=0 .
$$

The first of these (see MBHS) states simply that $\tilde{\eta}_{N}(\sigma)=1$ for $\sigma=-1$. Named "gyrostatic rigidity" by Poincare (1910), this result is unaltered by anelasticity and the presence of oceans. The second sum rule is mandated by the fact that $\left(1 / \tilde{\eta}_{R}(\sigma)\right)$ is proportional to $(e-\sigma)$.

Such violation means that the parameter estimates obtained with Fit II are not suitable for physical interpretation. Nevertheless, Fit II based on the resonance formula gives a very good representation of the VLBI-estimated nutation amplitudes (the discrepancies being less than three times the standard errors of the estimates, except for the 18.6 year amplitudes), which is useful for practical purposes.

The present study indicates what types of geophysical information may be obtained from nutations. Similar studies starting with various other VLBI-estimated nutation amplitude data sets need to be done before firm conclusions can be drawn regarding specific results.

\section{References}

Defraigne, P., Dehant, V., and Hinderer, J. (1994), J. Geophys. R., Vol. 99, pp. 92039213.

Dehant, V. (1990), Geophys. J. Int, Vol. 100, pp. 477-483.

Gwinn, C.R., Herring, T.A., and Shapiro, I.I. (1986), J. Geophys. R., Vol. 91, pp. 47454754.

Mathews, P.M., Buffett, B.A., Herring, T.A., and Shapiro, I.I. (1991), J. Geophys. R., Vol. 96, pp. 8219-8257.

Poincaré, H. (1910), Bull. Astron., Vol. 27, pp. 321-356.

Wahr, J.M., and Bergen, Z. (1986), Geophys. J. R. Astron. Soc., Vol. 87, pp. 633-668.

Zhu, S.Y., and Groten, E. (1989), Astron. J., Vol. 99, pp. 1024-1044. 